\title{
EVALUATION OF CLOSED CRACKS BY ANALYSIS OF SUBHARMONIC ULTRASOUND WITH CLOSURE STRESS
}

Kazushi Yamanaka, Tsuyoshi Mihara and Toshihiro Tsuji

Department of Materials Processing, Tohoku University, Japan

\begin{abstract}
Cracks in solids can be detected by ultrasound if they are open. However, their detection is not easy when they are closed with a closure stress, and it is a fundamental problem in ultrasonic testing. Subharmonics with half the input frequency is potentially useful in the detection and evaluation of such cracks. We developed analytical and numerical theories accounting for the crack parameters, such as closure stress and crack surface conditions, for the first time and proved their validity by comparison with experiments on a well-defined fatigue crack in aluminum alloy. Based on these theories, we propose a novel method to estimate size of partially closed cracks, which solves the fundamental problem in ultrasonic testing.
\end{abstract}

Introduction: Cracks in solids can be detected by ultrasound if they are open [1,2], since the ultrasound is reflected or scattered by the crack as shown in Fig.1(a). However, it is very difficult when the crack is closed since the ultrasound is transmitted as shown in (c). When the crack is partially closed with a closure stress, or by oxide films, as shown in Fig.1(b), the ultrasound is partially transmitted and the crack detection is not easy. This has been a fundamental problem in ultrasonic testing (UT) of cracks which many researchers have tried to solve without complete success.

To solve this problem, detection of superharmonic signals generated at cracks by the nonlinear effect of large amplitude ultrasound (Fig.1(d)), has been expected as the most promising approach[3-5]. However, the signal-to-noise $(\mathrm{S} / \mathrm{N})$ ratio of superharmonics is not very high, because it is generated also in piezoelectric transducers and liquid media, which becomes background noise. In particular, it is well known that the ultrasound characteristics in water, used in the liquid immersion UT, are highly nonlinear.

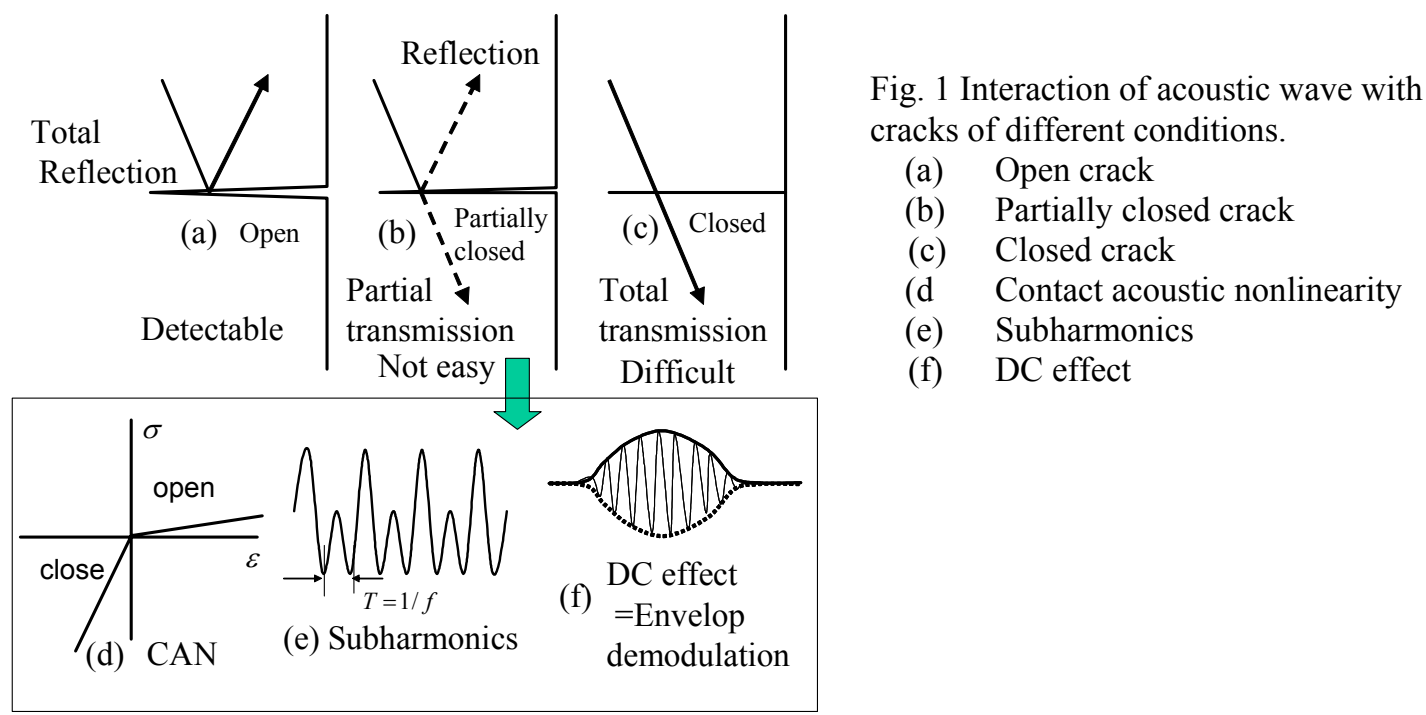

In this respect, subharmonics shown in Fig. 1 (e) [4,5] has much higher $\mathrm{S} / \mathrm{N}$ ratio than superharmonics and potentially useful for accurate sizing of partially closed cracks. Based on the theory of nonlinear contact vibration in atomic force microscopy [6,7], we developed a theory for subharmonic generation. Unlike others, it can reproduce experimentally observed subharmonic signals, employing the van der Waals interatomic force and its extension to interaction between larger objects [10]. It can account for the effect of crack parameters, such as closure stress and crack surface conditions [8], and proved their validity by comparison with experiments on welldefined fatigue cracks in aluminum alloy [9]. 
Theory: A partially closed crack is modelled in Fig. 2 (a). If a crack plane is divided into segments, each segment is represented by a mass and spring. The model of calculation is shown Fig.2 (b). Crack plane A is represented by the interaction force curve $F=f(x)$ vibrated as $a \sin \omega t$ resulting in $F=f(x-a \sin \omega t)$. Crack plane B is modelled by the mass $m$ at position $x(t)$ supported by a spring of stiffness $k$. To calculate subharmonic waveforms, we solve,

$m+2 x+k\left(x-x_{S}\right)=f_{0}\left[\kappa\left(\frac{\sigma}{x-a \sin \omega t}\right)^{\mathrm{M}}-\left(\frac{\sigma}{x-a \sin \omega t}\right)^{\mathrm{N}}\right]$

where, $x(t)$ is the position of crack plane $\mathrm{B}, f_{0}$ is the magnitude of force, $\mathrm{M}$ is the repulsive force index, $\mathrm{N}$ is the attractive force index, $\kappa$ is the weight of repulsive force relative to attractive force, and $\sigma$ is a characteristic length for crack planes (e.g., interatomic distance, grain size or asperity height, etc.). The parameter $x_{s}$ represents the equilibrium position of crack plane B without the interaction force $f(x)$, and a small value of $x_{s}$ corresponds to large closure stress. The parameters $\kappa, \mathrm{M}$ and $\mathrm{N}$ are given in the literature for various shapes of objects [10], as listed in Table 1. In this work, we assume that crack planes are a plane and a half space, and thus case (3) is employed. To simulate a semi-rigid wall with negligible attractive force, we let $\kappa=100$ in case (4).

\begin{tabular}{|l|l|l|l|}
\hline & $\mathrm{M}$ & $\mathrm{N}$ & $\kappa$ \\
\hline (1)Lennard-Jones atomic potential & 13 & 7 & 2 \\
\hline (2)Sphere- half space interaction & 8 & 2 & $1 / 30$ \\
\hline (3) Plane- half space interaction & 9 & 3 & 1 \\
\hline (4) Semi-rigid wall (empirical) & 9 & 3 & 100 \\
\hline
\end{tabular}

Table I Repulsive and attractive force index and weight of repulsive force.

The force towards $-x$ acted by the linear spring to crack plane B $F=k\left(x-x_{S}\right)$ is shown by the straight line which intersects with the $x$-axis at $x_{S}$. The intersection of curves $F=k\left(x-x_{S}\right)$ and $F=f(x)$ gives two equilibrium positions of crack plane B under the interaction force. In this example they are at around $\sigma$ and $10 \sigma$.
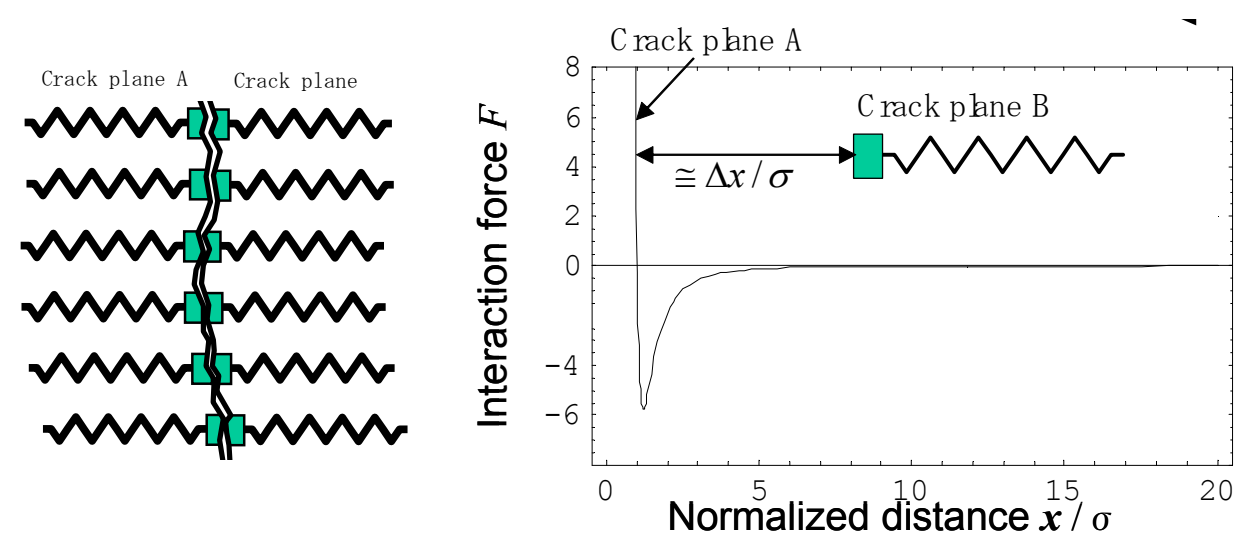

Figure 2. Model (a) A segment model of a partially closed crack (b) Two force acting to crack plane B

Potential energy of crack plane B in the field of linear spring and interaction potential is given by 
$U(x)=\frac{k\left(x-x_{S}\right)^{2}}{2}-f_{0}\left[\kappa \frac{1}{1-M}\left(\frac{\sigma}{x-x_{P}}\right)^{\mathrm{M}-1}-\frac{1}{1-N}\left(\frac{\sigma}{x-x_{P}}\right)^{\mathrm{N}-1}\right]$

where $x_{P}$ is position of crack plane A. Fig. 3(a) shows potential energy $U$ with $x_{P} / \sigma=-10,-4$, $0,4,10$, where $f_{0}=15$ and $k=0.2$ for case (4). The parabola represents the linear spring and the steep wall located at $x / \sigma \cong x_{P} / \sigma+2$ represents the repulsive force.

Since subharmonic generation is a strongly nonlinear phenomenon and is not fully understood, it is instructive to see how crack plane B behaves for an amplitude-modulated input signal. Fig. 3

(b) shows vibration waveform of crack plane B with the input amplitude $a / \sigma$ varied from 0 to 20 , where $m=1, \gamma=0.5$ and initial value of $x(t)=1.4 \sigma$.

When the frequency is low ( $\omega=0.5$ ), the crack moves to the equilibrium position at $x_{S} / \sigma=12$ (time A) and stays there. When the amplitude reaches $a / \sigma=10$, clapping starts and the crack starts to vibrate at frequency $\omega$ (time B). After that, the amplitude increases and decreases almost in proportional to the input wave amplitude. When the frequency is high $(\omega=1)$, the crack behaves similarly for low amplitudes. When the amplitude reaches $a / \sigma=10$, the clapping contact starts and the crack plane starts to vibrate at frequency $\omega / 2$ (time B) at subharmonic frequency. After that, the subharmonic amplitude increases. However, it then decreases at time $\mathrm{C}$ and after that no subharmonic signal appears at time $\mathrm{D}$, where the amplitude is reduced. Inset of lower figure (b) shows instance of clapping at 7 cycles before time $\mathrm{C}$, and it shows how the subharmonic signal decays.

It is interesting to note that for larger damping $(\gamma=1)$ the subharmonic signal survives at time $\mathrm{C}$ until time D (not shown here). From these results, it is shown that the subharmonic generation requires time delay due either to inertia (high $\omega$ ) or viscosity (large $\gamma$ ). Also it is noted that there is a hysteresis of subharmonic amplitude [5] since the waveform for $\omega=1$ is asymmetric.

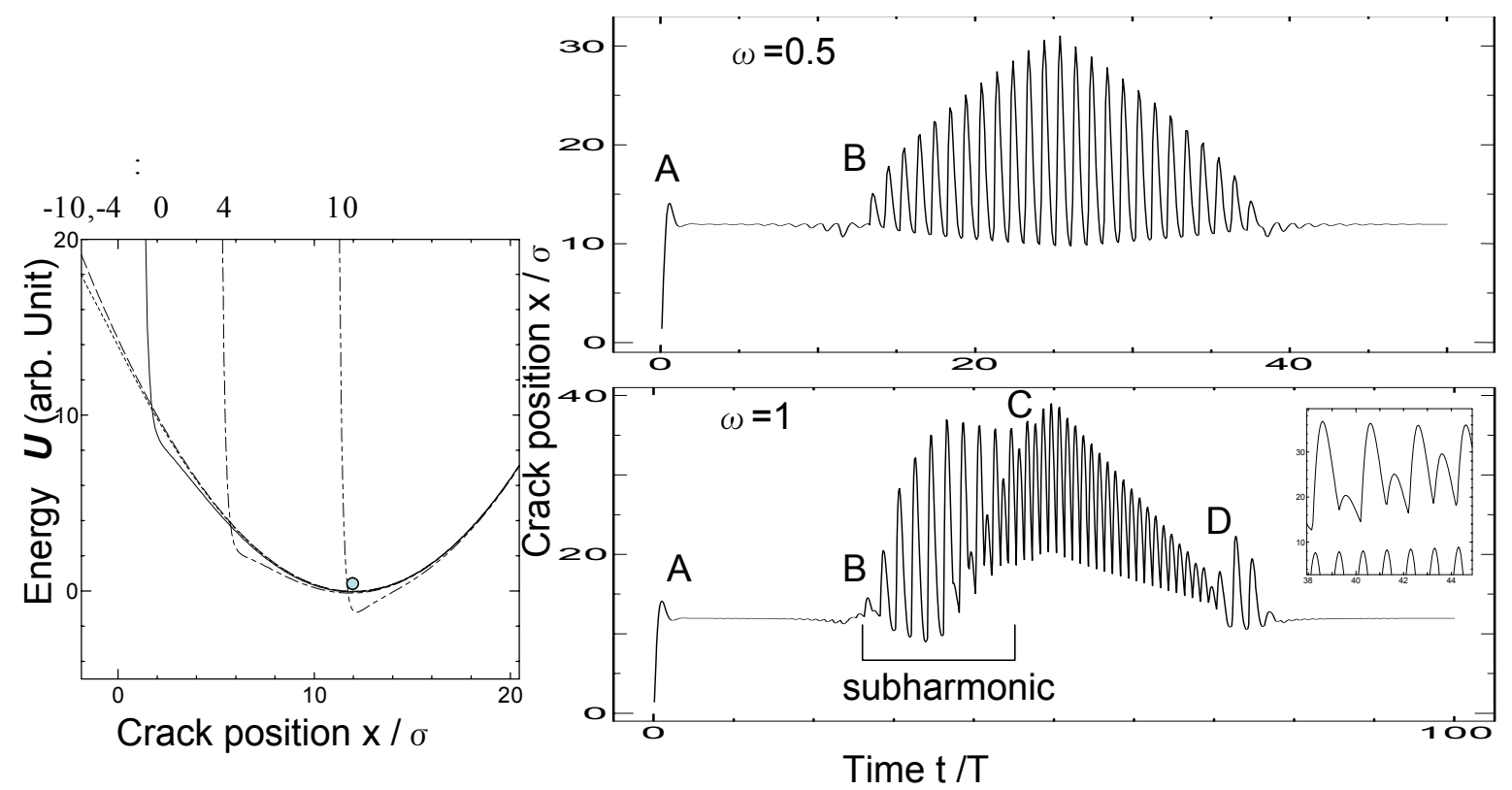

(a)

(b)

Fig. 3 Explanation of subharmonic generation. (a) Potential energy of crack plane B in the field of linear spring and interaction potential. (b) Vibration waveform of crack plane B due to amplitude modulated input signal. Inset of lower figure (b) shows instance of clapping contact at 7 cycles before time $\mathrm{C}$.

In usual testing, we propose to use constant amplitude tone burst with a few carriers, rather than an amplitude-modulated signal. The observed ultrasonic wave may be a kind of acoustic emission, 
which is generated by the variation of the crack opening displacement (COD) rather than a vibration of a single crack plane. Waveforms in Fig. 4 represent the COD $\Delta x=x(t)-a \sin \omega t$ normalized by the characteristic length $\sigma$ for the equilibrium position $x_{s}=10 \sigma$ and constant input wave amplitude of (a) $a=3 \sigma$, (b) $a=8 \sigma$ and (c) $a=10 \sigma$. Other parameters are $f_{0}=15$, $m=1, \gamma=0.5, k=0.2, \omega=1$, initial value of $x(t)=1.8 \sigma$. The waveform in (a) is a linear response with the power spectrum peak only at the input wave frequency $f$. Here, crack plane $\mathrm{B}$ is trapped at the equilibrium position close to $x_{s}=10 \sigma$. The observed vibration of COD is mainly due to the motion of crack plane A. The waveform in (b) appears to have a frequency of $f / 7$, but it is not exactly periodic (slightly chaotic), whereas that in (c) is periodic with a frequency of $f / 2$. This is a stable subharmonic wave. Here, crack plane B is sometimes lifted from the equilibrium position close to $x_{s}=10 \sigma$.

This calculation has important implication in the NDE of objects which may have partially closed cracks; If we find subharmonic waves when increasing the input wave amplitude, there is a partially closed crack, hidden in the object. Such a crack gives linear response when the input wave amplitude is small, which is similar to the response of objects without cracks or those with open cracks. Therefore, a capability of changing input wave amplitude in a wide range is essential for reliable NDE of objects with partially closed cracks.

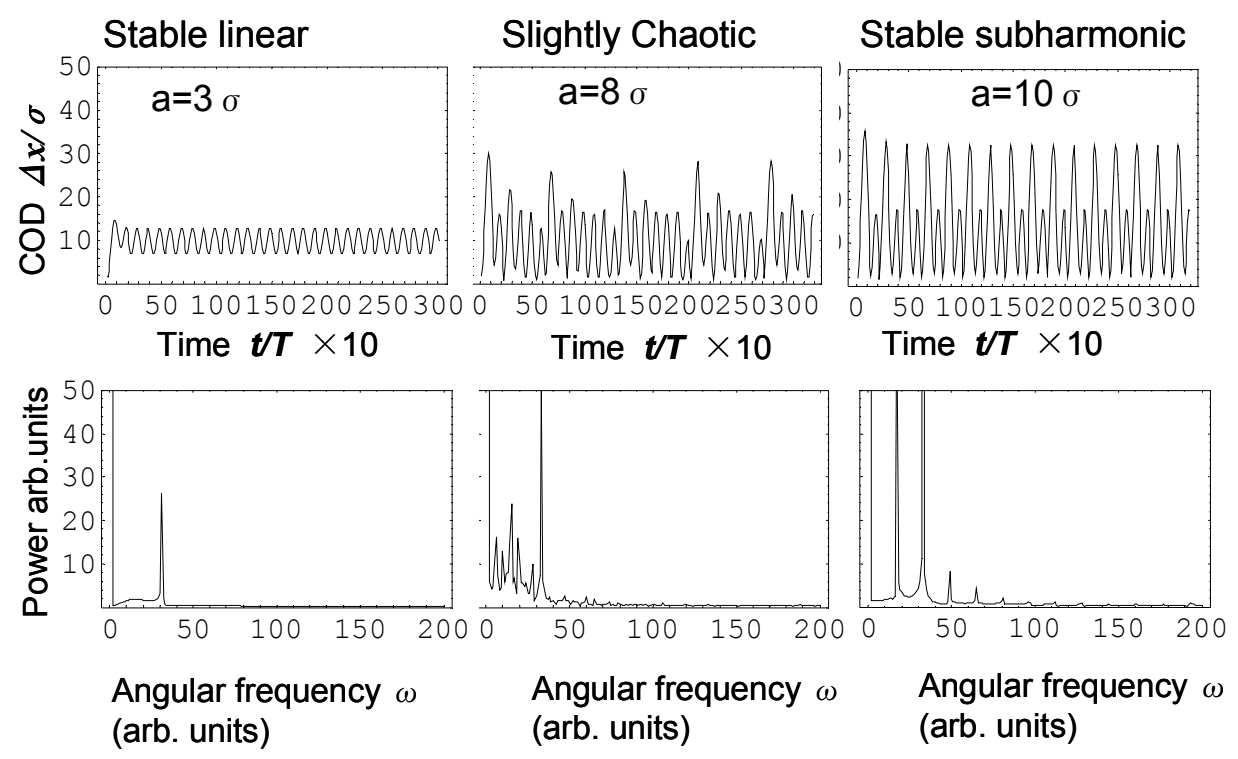

Figure 4. Calculated subharmonic waveforms with the parameters; $f_{0}=15, m=1, \gamma=0.5, k=0.2, \omega=1$, initial value of $x(t)=1.8 \sigma$. The equilibrium position of crack plane B $x_{s}=10 \sigma$

(a) Linear response with the input amplitude $a=3 \sigma$

(b) Chaotic response with the input amplitude $a=8 \sigma$

(c) Subharmonic wave with the input amplitude $a=10 \sigma$

Comparison with Experiment: In this section, we verify the theories in previous sections by using a fatigue crack in an aluminum alloy (A17030). The crack was extended from a notch in a bending fatigue test with the maximum stress intensity factor $K_{\max }=14 \mathrm{kgf} / \mathrm{mm}^{3 / 2}$ and the minimum stress intensity factor $K_{\min }=2 \mathrm{kgf} / \mathrm{mm}^{3 / 2}$. The crack tip echo from the oblique incidence longitudinal wave was monitored during the fatigue test, in order to confirm the arrival time variation of the echo when the stress intensity factor was varied between $K_{\max }$ and $K_{\min }$. The 
nonlinear ultrasonic measurement was performed using either a normal incidence or an oblique incidence longitudinal wave. For the oblique incidence, we employed a 45 degree polystyrene wedge.

A sinusoidal wave packet with 20 carriers at the frequency of $6.4 \mathrm{MHz}$ was amplified with a gated amplifier up to $1.2 \mathrm{kV}$. The displacement amplitude of the generated longitudinal wave was estimated using a laser interferometer. The amplitude of the observed waveform turned out to be larger than $40 \mathrm{~nm}$ peak to peak (p-p) in the case of normal incidence and $20 \mathrm{~nm}$ p-p in the case of oblique incidence, both at the position of the crack. For the detection, we used a broadband longitudinal wave transducer with the center frequency of $10 \mathrm{MHz}$.

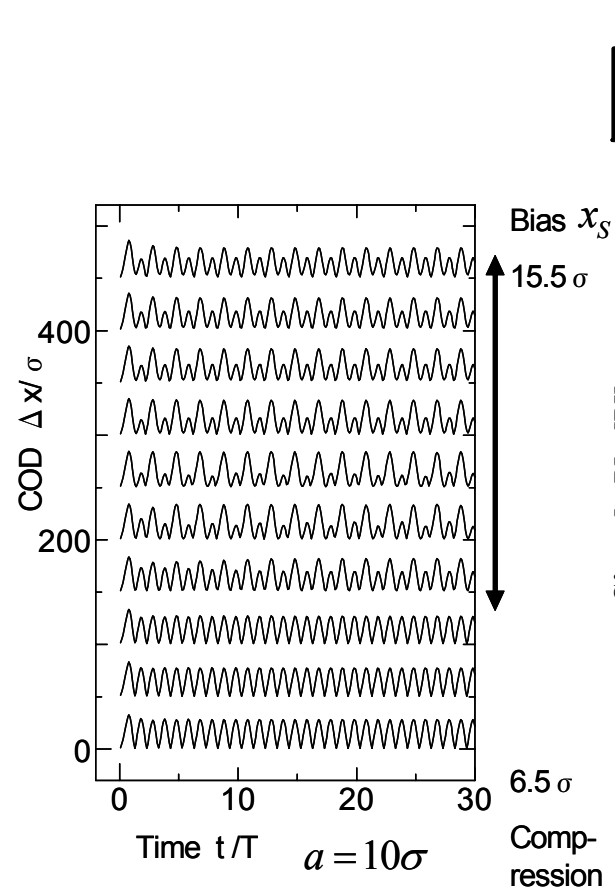

Calculated
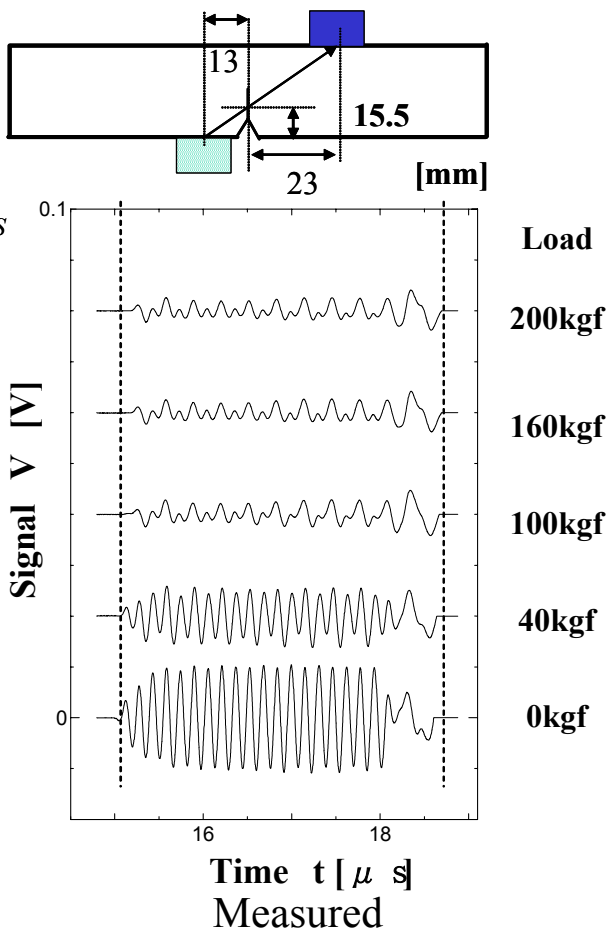

(a)

(b)

Figure 5. Effect of crack closure stress on the subharmonic generation: (a) calculated and (b) experimental waveforms in aluminium alloy fatigue crack specimen.

The crack was opened and closed by applying and removing a static bending load. The relationship between superharmonics and subharmonics and the crack closure was investigated. The waveforms (Fig. 5) are plotted with different bending loads in the oblique incidence test illustrated in the top figure. The amplitude of the input wave was measured to be $10.7 \mathrm{~nm}$ by using a Michelson-type laser interferometer. As the partially closed crack was opened by the bending load of up to $100 \mathrm{kgf}$, the amplitude of the echo decreased, and the amplitude of adjacent carriers became clearly different, resulting in the generation of subharmonics. It should be noted that the subharmonics was already evident within the first two carriers, showing that the temporal resolution could be made much higher than in CAN or conventional nonlinearity, where more than 10 carriers are required in order to obtain a sufficiently high $\mathrm{S} / \mathrm{N}$ ratio in the power spectra. In the corresponding power spectra, the intensities of $2 f$ and $3 f$ components were observed together with that of the subharmonic $f / 2$ component at $3.2 \mathrm{MHz}$. The subharmonic intensity was even larger than the fundamental intensity at bending loads above $100 \mathrm{kgf}$.

The amplitude dependence of the subharmonic intensity is verified both experimentally and theoretically in Fig. 6. 


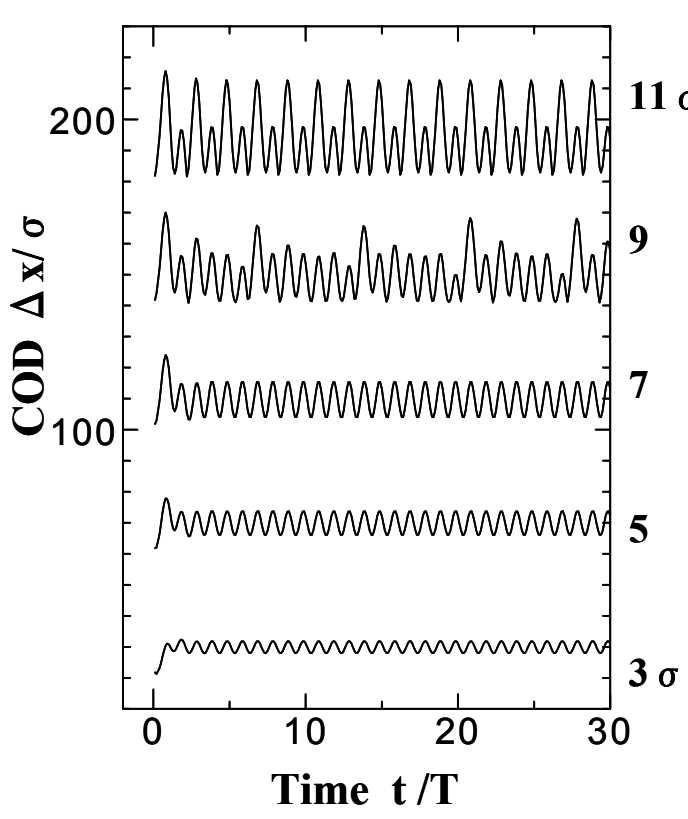

(a)

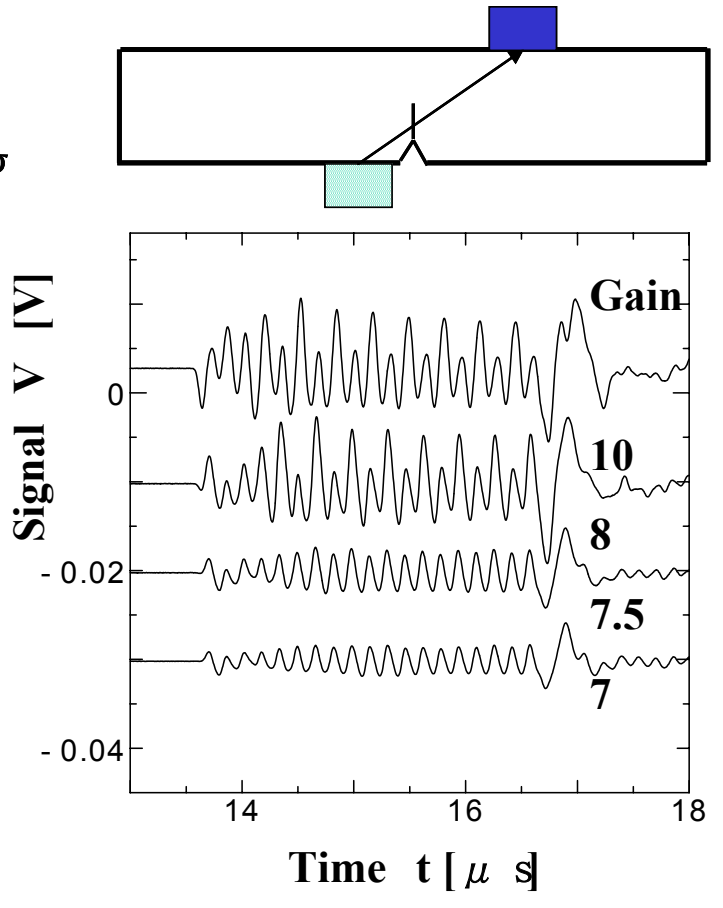

(b)

Figure 6. Effect of input wave amplitude on the subharmonic generation: (a) calculated and (b) experimental waveforms in aluminium alloy fatigue crack specimen.

Discussion: Although subharmonics have attracted considerable attention in the medical ultrasound, there is a significant difference in the physical process between the subharmonics generated at bubbles and at crack planes. The latter is closer to impact collision and the contact vibration at the solid surface, and it is simpler since the subharmonics is generated at only one position, and it is repetitive and coherent with the input wave.

There is a marked similarity between the theory of nonlinear crack vibration and the theory of nonlinear contact vibration in AFM. In fact, analytical equations explaining the DC effect and subharmonics were first derived to describe the demodulation of high frequency vibration in the ultrasonic force microscopy, which is a preliminary version of AFM realizing elasticity evaluation of stiff materials that conventional AFM cannot realize[11-15].
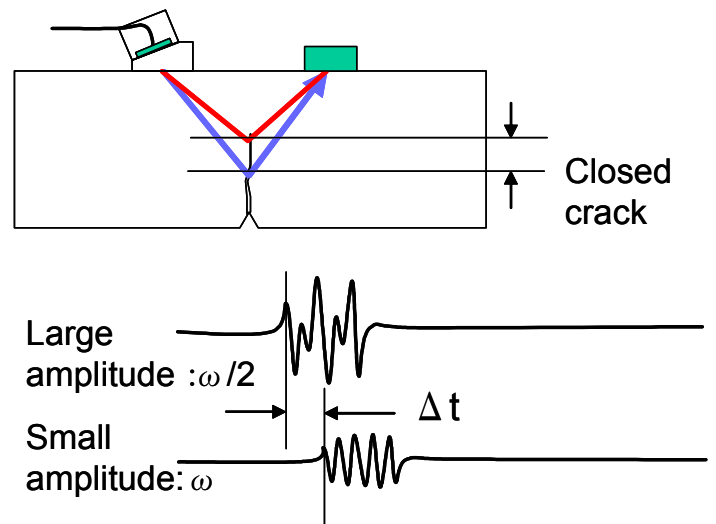

\section{Number of $\quad \Delta t$ : Advance of echo due to waves 4-6 opening of closed crack}

Figure 7 Accurate sizing of partially closed crack by time of flight of subharmonic wave 
The major application of observing subharmonic signal is to improve the accuracy of crack sizing, especially where the accuracy is insufficient due to crack closure caused by residual stresses or interfacial oxide films. The depth of partially closed crack can be determined in a configuration shown in Fig. 7 as

$d=\sqrt{(V t / 2)^{2}-l^{2}}-\sqrt{[V(t-\Delta t) / 2]^{2}-l^{2}}$

where $l$ is the lateral distance between the transducer and crack, $t$ is the propagation time of the linearly scattered wave of weak input wave, $\Delta t$ is the propagation time difference between the linearly scattered wave of weak input wave and the subharmonic wave generated by the strong input wave and $V$ is velocity of ultrasound. When the crack has irregular shape, as is the case for stress corrosion cracks, phased array is employed after filtering at subharmonic frequencies. Such a novel equipment is being development and it will significantly reduce the sizing error problem in aged atomic power plants.

Conclusions: Subharmonics generated at partially closed cracks are potentially useful to detect cracks which are not easy to detect by conventional ultrasonic testing or superharmonics in nonlinear ultrasound. In this work, we developed analytical and numerical theories accounting for the crack parameters such as closure stress and crack surface conditions, for the first time, and prove their validity by comparison with experiments on well-defined fatigue cracks in aluminum alloy. It will be possible to estimate the size, closure stress and physical properties of partially closed crack planes by comparing measured waveforms and theoretical predictions. We expect this approach to be useful for solving the fundamental problem in the ultrasonic testing of cracks.

This work was supported by a Grant-in-Aid for Science Research (No. 15656179,16206071) from the Ministry of Education, Culture, Sports, Science and Technology.

\section{References:}

1) L.M. Schmerr, Jr.:Fundamentals of Ultrasonic Nondestructive Evaluation (Plenum Press New York 1998) pp305-383.

2) K. Yamanaka and Y. Enomoto: J. Appl. Phys. 53 (1982) 846.

3) O. Buck, W.L. Morris and J.M. Richardson: Appl. Phys. Lett. 33 (1978) 271.

4) B.A.Korshak, I.Yu. Solodov and E.M. Ballad: Ultrasonics 40 (2002) 707.

5) A. Moussatov, V. Gusev and B. Castagnede: Phys. Rev. Lett. 90 (2003) 124301

6) K. Yamanaka, H. Ogiso and O. Kolosov: Appl. Phys. Lett. 64 (1994) 178.

7) K. Yamanaka: Advances in Acoustic Microscopy, Volume 1, ed. Andrew Briggs (Plenum Press, New York, 1995). p 301.

8) Kazushi Yamanaka, Tsuyoshi Mihara and Toshihiro Tsuji, Jpn. J. Appl. Phys. 43 (2004) 3082.

9) M. Akino, T. Mihara and K. Yamanaka: Rev. Prog. QNDE 23B (2004) 1256.

10) D. Maugis: Contact, Adhesion and Rupture of Elastic Solid (Springer , 1999) ..

11) K. Yamanaka and S. Nakano: Jpn. J. Appl. Phys. 35 (1996) 3787.

12) U. Rabe and W. Arnold: Rev. Sci. Instrum. (1996).

13) T. Tsuji and K. Yamanaka: Nanotechnology 12 (2001) 301.

14) T. Tsuji, H. Irihama and K. Yamanaka: Jpn. J. Appl. Phys. 41 (2002) 832.

15) T. Tsuji, H. Ogiso, J. Akedo, S. Saito, K. Fukuda and K. Yamanaka: Jpn. J. Appl. Phys. 43 (2004). 